\title{
Ueber die Anzahl der willkürlichen Constanten in algebraischen Functionen.
}

\author{
(Von Herrn G. Roch in Halle.)
}

Ist $s$ eine durch die Gleichung $F(s, z)=0$ definirte algebraische Function von $z$, so kann nach Riemann (s. dessen Abhandlung über Abelsche Functionen, Band 54. dieses Journals) jede wie $s$ verzweigte algebraische Function $s^{\prime}$ von $z$ rational durch $s$ und $z$ ausgedrückt werden. Wird die Function $s^{\prime}$ in $m$ Punkten der Fläche $T$, welche die Verzweigungsart angiebt, unendlich erster Ordnung, so enthält dieselbe nach $\$$. 5. der erwähnten Abhandlung $m-p+1$ willkürliche Constanten. Schon die a. a. 0. untersuchte Bedingung der Existenz von Functionen, die in weniger als $p+1$ Punkten unendlich werden, zeigt, dass die Anzahl der wirklich vorhandenen Constanten eine grössere sein kann. Dies kann aber auch Statt finden, wenn $m$ grösser als $p$ ist. Ist z. B. $s^{\prime}$ der Quotient zweier Functionen $\varphi$, so wird $s^{\prime}$ in den $2 p-2$ Punkten unendlich, in denen der Nenner gleich Null ist (s. \$. 10. der citirten Abhandlung), und enthält so viele willkürliche Constanten, als die den Zähler bildende Function, nämlich $p$, während die Zahl $m-p+1$ im vorliegenden Falle gleich $\boldsymbol{p}-1$ ist. Sind dagegen $\varphi_{1}, \varphi_{2}, \psi_{1}, \psi_{2}$ solche Functionen $\varphi$, welche in $p-1$ Punkten unendlich klein zweiter Ordnung werden, deren Quadratwurzeln Riemann Abelsche Functionen nennt, so giebt es eine gewisse Anzahl von Ausdrücken $\sqrt{\frac{\Psi_{1} \psi_{1}}{P_{2} \psi_{2}}}$, welche rationale Functionen von $s$ und $z$ sind; diese enthalten in der That $p-1$ Constanten in linearer Weise, ein Satz, der von Riemann herrührt und für welchen ein Beweis in der folgenden genauen Bestimmung der Constanten-Anzahl mit enthalten ist.

Der allgemeinste Ausdruck eines Integrals zweiter Gattung, welches in $m$ Punkten $\varepsilon$ unendlich erster Ordnung wird, ist

$$
v=\beta_{1} t_{1}+\cdots+\beta_{m} t_{m}+\alpha_{1} w_{1}+\cdots+\alpha_{p} w_{p}+\text { const. }
$$

(Vergl. \$. 5. der Riemannschen Abhandlung.) Hierbei sind unter $t_{1} \ldots t_{m}$ specielle Integrale zweiter Gattung zu verstehen, welche beziehlich in den Punkten $\varepsilon_{1} \ldots \varepsilon_{m}$ unendlich werden wie $\frac{1}{\sigma_{1}} \cdots \frac{1}{\sigma_{m}}$, wenn $\sigma_{k}$ eine Grösse be- 
deutet, die in $\varepsilon_{k}$ unendlich klein erster Ordnung genannt wird. Damit $v$ in eine algebraische Function $s^{\prime}$ übergehe, müssen sämmtliche Periodicitätsmoduln von $v$ verschwinden. Hieraus ergeben sich $2 p$ lineare Bedingungsgleichungen zwischen den $m+p$ Constanten $\beta$ und $\alpha$, so dass $m-p+1$ willkürliche Constanten bleiben. Nur, wenn diese $2 p$ Gleichungen von einander abhängig sind, enthält $s^{\prime}$ mehr als $m-p+1$ Constanten.

Um diese Abhängigkeit zu untersuchen, nehmen wir für die endlich bleibenden Integrale $w_{1} \ldots w_{p}$ die speciellen

$$
u_{1}=\int \frac{\varphi_{1}(\boldsymbol{s}, \boldsymbol{z}) d z}{\frac{\partial \boldsymbol{F}}{\partial \boldsymbol{s}}}, \ldots u_{p}=\int \frac{\varphi_{p}(\boldsymbol{s}, \boldsymbol{z}) d z}{\frac{\partial \boldsymbol{F}}{\partial \boldsymbol{s}}},
$$

welche Riemann als Argumente der $\vartheta$-Function einführt. Das Integral $u_{\mu}$ hat an dem Querschnitte $\left(a_{\mu}\right)$ den Periodicitätsmodul $\pi i$, an den übrigen $p-1$ Querschnitten $(a)$ die Periodicitätsmoduln Null, und an dem Querschnitt $\left(b_{\nu}\right)$ den Periodicitätsmodul $a_{\mu, \nu}$. In der Nähe der Punkte $\varepsilon$ gilt die Entwickelung

$$
\text { (a.) } \quad \boldsymbol{u}_{\mu}=\boldsymbol{u}_{\mu}\left(\varepsilon_{k}\right)+\boldsymbol{a}_{\mu}^{(k)} \sigma_{k}+b_{\mu}^{(k)} \sigma_{k}^{2}+\cdots
$$

Im Allgemeinen, d. h. wenn $\varepsilon_{1} \ldots \varepsilon_{m}$ weder im Unendlichen liegen noch Verzweigungspunkte sind, ist

$$
\boldsymbol{a}_{\mu}^{(k)}=\frac{\boldsymbol{\varphi}_{\mu}\left(\boldsymbol{s}_{k}, \boldsymbol{z}_{k}\right)}{\frac{\partial \boldsymbol{F}\left(\boldsymbol{s}_{k}, \boldsymbol{z}_{k}\right)}{\partial \boldsymbol{s}_{k}}} .
$$

Die Integrale zweiter Gattung $t_{1} \ldots t_{m}$ könnnen wir uns so gewählt denken, dass ihre Periodicitätsmoduln an den $p$ Querschnitten $(a)$ verschwinden. Denn gesetzt, $t_{k}$ hätte an den Querschnitten $\left(a_{1}\right), \ldots\left(a_{p}\right)$ die Periodicitätsmoduln $\tau_{k, 1}, \ldots \tau_{k, p}$, so hat man nur $t_{k}=t_{k}^{\prime}+\frac{1}{\pi i}\left(\tau_{k, 1} u_{1}+\cdots+\tau_{k, p} u_{p}\right)$ einzuführen, damit $t_{k}$ die angegebene Eigenschaft besitze. Dann reduciren sich die Bedingungen dafür, dass die Periodicitätsmoduln des Ausdruckes

$$
s^{\prime}=\beta_{1} t_{1}+\cdots+\beta_{m} t_{m}+\alpha_{1} u_{1}+\cdots+\alpha_{p} u_{p}+\text { const. }
$$

an den Querschnitten $(a)$ verschwinden, auf die Gleichungen

$$
\alpha_{1} \pi i=0, \quad \alpha_{2} \pi i=0, \quad \ldots \quad \alpha_{p} \tau_{i}=0 .
$$

Die übrig bleibenden Constanten $\beta$ sind nur noch den $p$ Bedingungsgleichungen für das Verschwinden der Periodicitätsmoduln an den Querschnitten (b) unterworfen, welche im Folgenden entwickelt werden sollen.

Das ganze Integral $\int \dot{u}_{\mu} d v$ durch die Begrenzung der Fläche $T^{\prime}$ (d. i. die durch die Querschnitte $(a)$ und $(b)$ einfach zusammenhängend gemachte 
Fläche $T$ ) erstreckt, ist gleich $\int\left(u_{+}-\underline{u}_{\mu}\right) d v$, unter $\underline{+}_{+}$und $\underline{u}_{\mu}$ die Werthe von $u_{\mu}$ auf den positiven und auf den negativen Seiten der Querschnitte verstanden und letzteres Integral positiv nur durch die positiven Seiten aller Querschnitte erstreckt. Dies ergiebt als Betrag des Integrales, analog wie in $\$ .20$ der Riemannschen Abhandlung:

$$
\pi i B_{\mu}-\sum_{\nu=1 \ldots p} A_{\nu} a_{\mu, \nu}
$$

wo $A$ und $B$ die Periodicitätsmoduln von $v$ an den Querschnitten $(a)$ und $(b)$ bezeichnen. - Andrerseits ist das Integral $\int \dot{u}_{\mu} d v$ gleich der Summe der um die Punkte $\varepsilon$ herum erstreckten Integrale, wobei, wenn einer der Punkte ein $(n-1)$ facher Verzweigungspunkt ist, die Curve, über welche um diesen Punkt herum integrirt wird, $n$ ganze Umläufe machen muss, um geschlossen zu sein. Auf diese Weise gelangt man zu der Gleichung

$$
\text { (1.) } \quad \pi i B_{\mu}-\sum_{\nu=1 \ldots p} A_{\nu} a_{\mu, \nu}=-2 \pi i \sum_{k=1 \ldots m} \beta_{k} a_{\mu}^{(k)},
$$

welche, da " die Werthe $1,2, \ldots p$ annehmen kann, $p$ Gleichungen repraesentirt und die Abhängigkeit der Periodicitätsmoduln $A$ und $B$ von der Lage der Punkte $\varepsilon$ enthält.

Im Folgenden sei der Einfachheit wegen vorausgesetzt, dass alle Punkte $\varepsilon$ im Endlichen liegen und nicht Verzweigungspunkte sind. Dann geht die Gleichung (1.) über in

$$
\text { (2.) } \quad \pi i B_{\mu}-\sum_{\nu=1 \ldots p} A_{\nu} a_{\mu, \nu}=-2 \pi i \sum_{k=1 \ldots m} \frac{\beta_{k} \varphi_{\mu}\left(s_{k}, z_{k}\right)}{\frac{\partial F\left(s_{k}, z_{k}\right)}{\partial s_{k}}} .
$$

Mit Hülfe dieser Formel verwandeln sich die oben erwähnten $p$ Gleichungen $B_{1}=0, B_{2}=0, \ldots B_{p}=0$, da die Periodicitätsmoduln $A$ im vorliegenden Falle gleich Null sind, in

$$
\sum_{k=1 \ldots m} \frac{\beta_{k} \varphi_{\mu}\left(s_{k}, z_{k}\right)}{\frac{\partial \boldsymbol{F}\left(\boldsymbol{s}_{k}, z_{k}\right)}{\partial \boldsymbol{s}_{k}}}=0 \text { für } \quad \boldsymbol{u}=1,2 \ldots p .
$$

Von diesen $p$ Gleichungen wird immer und auch nur dann eine eine Folge der übrigen, wenn sich $p$ Coefficienten $c_{1}, \ldots c_{p}$ in $\varphi=c_{1} \varphi_{1}(s, z)+\cdots+c_{p} \varphi_{p}(s, z)$ so bestimmen lassen, dass $\frac{\varphi\left(s_{k}, \bar{z}_{k}\right)}{\frac{\partial \boldsymbol{F}\left(\boldsymbol{s}_{k}, \boldsymbol{z}_{k}\right)}{\partial s_{k}}}$ gleich Null ist für jeden der $m$ Werthe von $k$, oder wenn die $m$ Punkte $\varepsilon$ solche sind, in denen ausser den $r$ zusammengefallenen sich aufhebenden Verzweigungspunkten eine Function $\varphi$ ver- 
schwinden kann. Ebenso können zwei oder mehrere von den $p$ Gleichungen aus den übrigen folgen und man kann das allgemeine Resultat in folgender Weise aussprechen:

Wird eine Function $s^{\prime}$ in $m$ Punkten unendlich gross erster Ordnung und können in diesen $m$ Punkten $q$ Functionen $\frac{\varphi(s, z)}{\frac{\partial F}{\partial s}}$ verschwinden, zwischen denen keine lineare Gleichung mit constanten Coefficienten besteht, so enthält $s^{\prime}$ die Zahl $m-p+1+q$ willkürlicher Constanten.

Sei z. B. $F(s, z)=0$ die Gleichung einer Curve fünfter Ordnung. Dann ist im Allgemeinen, d. h wenn die Curve keinen Doppelpunkt besitzt, $p=6$; die endlich bleibenden Integrale sind:

$$
\int \frac{a s^{2}+b s z+c z^{2}+d s+e z+f}{\frac{\partial F}{\partial s}} d z
$$

also jede ganze Function zweiten Grades ist eine Function $\varphi$. Die Function

$$
s^{\prime}=\frac{a s+b z+c}{a_{1} s+b_{1} z+c_{1}}
$$

wird in den 5 Punkten unendlich, in denen die Gerade $a_{1} s+b_{1} z+c_{1}=0$ die gegebene Curve schneidet. In diesen 5 Punkten verschwinden 3 von einander linear unabhängige Functionen $\varphi$, nämlich: $a_{1} s+b_{1} z+c_{1},\left(a_{1} s+b_{1} z+c_{1}\right) s$ und $\left(a_{1} s+b_{1} z+c_{1}\right) z$. Daher enthält $s^{\prime}$ die Anzahl $5-6+1+3=3$ willkürlicher Constanten, in der That die $a, b, c$.

Hat die gegebene Curve fünften Grades einen Doppelpunkt, so ist $p=5$. Dann sind, wenn $g=0, h=0$ zwei durch den Doppelpunkt gehende Gerade bedeuten, nur $\left(a_{1} s+b_{1} z+c_{1}\right) g$ und $\left(a_{1} s+b_{1} z+c_{1}\right) h$ Functionen $\varphi$, die in den 5 Punkten Null sind, in denen $s^{\prime}$ unendlich ist und $s^{\prime}$ enthält wiederum $5-5+1+2=3$ Constanten.

Liegen die Punkte $\varepsilon$ theilweise im Unendlichen, so muss man nach der Anzahl von Functionen $\varphi$ fragen, die von niedrigerem als dem höchst möglichen Grade sind und in den Punkten $\varepsilon$ verschwinden, die im Endlichen liegen.

Bei ganz .allgemeiner Lage der Punkte $\varepsilon$ lässt sich die Regel über die Anzahl der Constanten nur so fassen: Sind $c_{1} \ldots c_{p}$ zu bestimmende Constanten und sind die endlich bleibenden Integrale durch die Reihen (a) dargestellt, so enthält eine algebraische Function, die in $m$ Punkten unendlich erster Ordnung ist, $m-p+1+q$ wilkürliche Constanten, wenn es $q$ nicht linear von einander 
abhängige Functionen

$$
c_{1} a_{1}+\cdots+c_{p} a_{p}, \quad a_{v}=\frac{d u_{v}(s, z)}{d \sigma}
$$

giebt, welche in den Punkten $\varepsilon$ verschwinden.

Für die Bestimmung der Functionen, die in einzelnen Punkten unendlich von höherer Ordnung werden, kann man entweder in dem Vorigen Punkte $\varepsilon$ auf einander fallen lassen oder die Betrachtungen selbstständig anstellen durch Benutzung der Coefficienten höherer Potenzen von $\sigma$ in den Reihen (a).

Die Gleichungen (1.) enthalten Resultate für die ganzen Integrale zweiter Gattung, welche von Weierstrass für hyperelliptische Integrale schon früher gegeben worden sind.

Halle, 1864. 